\title{
Téoros
}

Revue de recherche en tourisme

\section{Quelques considérations sur les conditions de travail des femmes dans le secteur de l'hébergement et de la restauration}

\section{Lise Poulin et François Aubry}

Volume 16, numéro 3, automne 1997

Femmes et tourisme

URI : https://id.erudit.org/iderudit/1073313ar

DOI : https://doi.org/10.7202/1073313ar

Aller au sommaire du numéro

Éditeur(s)

Université du Québec à Montréal

ISSN

0712-8657 (imprimé)

1923-2705 (numérique)

Découvrir la revue

Citer cet article

Poulin, L. \& Aubry, F. (1997). Quelques considérations sur les conditions de travail des femmes dans le secteur de l'hébergement et de la restauration.

Téoros, 16(3), 19-20. https://doi.org/10.7202/1073313ar 


\section{QuelQues CONSIDÉRATIONS SUR LES CONDITIONS DE TRAVAIL DES FEMMES DANS LE SECTEUR DE L'HÉBERGEMENT ET DE LA RESTAURATION}

Lise Poulin, présidente

Fédération du commerce, CSN

François Aubry, économiste

Service de la recherche, CSN

En 1994, au Québec, 34400 personnes travaillaient dans le secteur de l'hébergement et 155800 dans celui de la restauration. Environ $60 \%$ de ces emplois étaient détenus par des femmes. Ensemble, ces deux secteurs regroupent les deux tiers de tous les emplois apparentés à l'industrie touristique.

Comme c'est souvent le cas dans cette industrie, une grande partie des emplois dans les secteurs de l'hébergement et de la restauration sont précaires, c'est à dire à temps partiel, saisonniers, temporaires et non syndiqués. Citons quelques données propres à illustrer ce phénomène. En $1994,25 \%$ des employé(e)s du secteur de l'hébergement ont travaille à temps partiel et $28 \%$ ont travaille moins de 30 semaines au cours de l'année; dans la restauration, ces pourcentages étaient de $35 \%$ et de $28 \%$ respectivement.

Outre la précarité, d'autres facteurs affectent plus particulièrement la qualité de vie au travail des femmes qui oeuvrent dans ces secteurs. Bien que certaines formes de discrimination et d'iniquité aient été éliminées, les travailleuses font toujours l'objet de comportements, de pratiques et d'attitudes à caractère sexiste ou discriminatoire. Nous en décrirons brièvement les principaux.

\section{DISCRIMINATION SALARIALE}

Les femmes qui travaillent dans les secteurs de l'hébergement et de la restauration ont souvent été victimes de discrimina- tion salariale. Des progrès ont tout de même été accomplis. En voici quelques exemples.

Jusqu'au milieu des années 1980, pour un travail équivalent, les préposées aux chambres recevaient un salaire moindre que celui des équipiers ou des préposés à la buanderie, qui étaient en grande majorité des hommes. La négociation collective a permis d'éliminer ces différences salariales, mais elles persistent dans les milieux de travail non syndiqués.

Dans le secteur de l'hôtellerie, les emplois de cuisinier et d'aide cuisinier sont historiquement occupés en grande majorité par des hommes. Or, les salaires attachés à ces occupations sont généralement supérieurs à ceux versés dans d'autres départements. Ainsi, depuis quelques années, de plus en plus de femmes effectuent un rattrapage en accédant à ces postes de travail mieux rémunérés, ceci souvent après avoir suivi une formation appropriée à l'Institut de l'hôtellerie et de la restauration.

Mais malgré des améliorations aux conditions salariales des femmes, les iniquités sont encore nombreuses. Une étude du
Groupe DBSF indique qu'il existe pour certains métiers ou corps d'emplois des différences parfois substantielles entre le salaire des femmes et celui des hommes. En voici quelques exemples tirés de cette étude.

\begin{tabular}{|l|l|l|}
\hline Métier & Hommes & Femmes \\
\hline Gouvernance d'ho̊tel & $30144 \$$ & $18554 \$$ \\
\hline Maittre d'hôtel & 21445 & 12233 \\
\hline Réceptionniste d'hôtel & 18261 & 14888 \\
\hline Aide cuisinier & 15261 & 14813 \\
\hline
\end{tabular}

Toujours dans le secteur de l'hébergement, le métier de préposée à la réception, occupé en grande majorité par des femmes, est sous rémunéré si on le compare à celui de préposé à l'entretien général, poste détenu surtout par des hommes; les qualifications exigées pour le deux corps d'emploi sont pourtant équivalentes. Les travailleuses comptent bien recourir à la Loi sur l'équité salariale adoptée en novembre 1996 pour venir mettre fin à ces injustices.

\section{DES HORAIRES DE TRAVAIL CASSE-TÊTE}

Les horaires de travail du secteur de la restauration et des bars sont souvent atypiques. Non seulement y retrouve-t-on beaucoup d'emplois à temps partiel mais plusieurs salarié(e)s travaillent de soir ou de nuit, et souvent selon un horaire d'heures brisées. À titre d'exemple, il est courant qu'une serveuse de restaurant travaille de 11 heures du matin à 2 heures de l'après-midi, puis de 5 de l'après-midi à 8 ou 9 heures du soir. Comme on peut 
l'imaginer, ce type d'horaire rend très difficile, voire impossible, la conciliation des responsabilités professionnelles et familiales, particulièrement pour les femmes chefs de famille monoparentale.

Des problemes analogues se posent dans le secteur de l'hôtellerie, cette fois à cause de régimes de travail qui obligent un certain nombre de préposées aux chambres à se présenter sur appel. Ainsi, ces femmes ne savent qu' à la toute dernière minute si elles travailleront ou non durant la journée. Dans ces conditions, organiser la garde des enfants devient extrểmement problématique.

\section{TRAVAILLEUSES A POURBOIRE}

La majorité des personnes dont une partie de la rémunération est versée sous forme de pourboires sont des femmes. II est généralement reconnu aujound' hui que les travailleuses et les travailleurs à pourboire font face ầ un problème d'équitế en ceci que aleurs bénéfices sociaux (assurance emploi, régime de rentes, paie de vacances) sont calculés à partir d'un revenu qui exclut les pourboires, bien qu'ils soient partie intégrante de leurs revenus imposables\%. Des pourparlers sont actuellement en cours entre le Gouvernement du Québec, I'Association des restaurateurs et les organisations syndicales afin de trouver une solution à ce probleme.

\section{DES ATTITUDES SEXISTES}

Il existe aussi dans les secteurs de l'hếbergement et de la restauration d'autres problèmes de discrimination et d'iniquités qui cette fois relèvent de comportements ou d'attitudes sexistes ou racistes.

Par exemple, il arrive encore que certains employeurs evitent d'embaucher des membres des minorités visibles pour des postes où ils seraient en relation directe avec la clientèle, tels serveuse, serveur ou préposé(e) à la réception. Ils préfèrent faire travailler ces personnes en coulisse ou en arrière scène.

Il arrive aussi que des membres de communautés culturelles se sentent discriminés à l'embauche à cause de leur difficulté à maîtriser la langue française. Des efforts avaient pourtant été faits au début des an- nées 1990 pour remédier à cette situation. En effet, à la demande de la Fédération du commerce (CSN), des cours de français avaient été mis sur pied conjointement par les employeurs et les syndicats pour des employé(e)s du secteur de l'hôtellerie. Certaines de ces expériences se poursuivent aujourd'hui, mais d'autres ont malheureusement dô être abandonnées pour diverses raisons.

Les travailleuses et les travailleurs âgés peuvent aussi être victimes de discrimination. Comme c'est le cas pour certains membres des minorités visibles, on les Écartera souvent de certains postes de travail où le contact avec la clientèle est fréquent. On préférera embaucher des travailleuses et des travailleurs plus jeunes et de belle apparence. Comme on le sait, de telles pratiques sont relativement courantes dans plusieurs secteurs de services aux personnes.

On peut aussi trouver des exemples de discrimination dans le milieu très particulier de la grande restauration ou des clubs privés. Dans ces milieux, il subsiste souvent une vieille tradition qui veut que seuls des hommes ont la dignité requise pour servir une certaine clientèle, le plus souvent masculine et très à l'aise. La syndicalisation d'une partie de cette maind'oeuvre a permis de mettre fin à de telles pratiques discriminatoires dans un certain nombre d'établissements. Mais elle $n^{5}$ a pas pour autant modifié la mentalité sexiste d'une partie de la clientèle et de certains employeurs pour qui la recherche du gain monétaire passe avant les principes de justice et d'équité.

Les bars et les restaurants sont des lieux où des employées ou des clientes peuvent être victimes d'expériences désagréables, allant de quolibets sexistes et de remarques désobligeantes au harcèlement sexuel. La Commission des droits de la personne du Québec souligne dans plusieurs de ses rapports d'enquête qu'une proportion importante des plaintes pour harcèlement sexuel provient du milieu de la restauration. On remarque aussi chez certains restaurateurs une tendance à vouloir attirer une clientèle particulière en mettant l'accent sur l'apparence physique des serveuses dans leur publicité. Ceci n'est pas pour décourager le harcèlement.

Des conditions particulières de travail peuvent engendrer chez certaines catégo- ries d'employé(e)s un stress lié à une inquiétude très compréhensible quant à la protection de leur intégrité physique. C’est le cas en particulier pour les préposées aux chambres dans les hôtels et les motels qui travaillent souvent sans protection dans des lieux isolés; cette situation les rend vulnérables aux agressions physiques. Soulignons que de tels incidents se sont déjà produits au Québec. Les syndicats tentent de négocier diverses mesures de prévention, comme l'obligation pour l'employeur de fournir à ces employées un avertisseur sonore portatif ou l'instauration d'équipes formées de deux préposées aux chambres travaillant toujours ensemble.

\section{CONCLUSION}

Par ces quelques exemples, nous avons voulu démontrer que les femmes qui oeuvrent dans les secteurs de l'hébergement et de la restauration sont exposées à des formes particulières de discrimination ou subissent des préjudices qui sont liés aux conditions et à l'organisation de leur travail. Certaines relèvent de pratiques patronales insouciantes ou discriminatoires alors que d autres trouvent leur source dans des attitudes et des comportements sexistes toujours bien ancrés dans notre culture, ceci malgré des années de revendication féminine. Sans être une panacée, l'accélération de la syndicalisation de ces employé(e)s constitue un moyen privilégié pour ameliorer leur situation. Encore faudra-t-il que nos lois du travail soient amendées afin de permettre un important mouvement de syndicalisation.

\section{BIBLIOGRAPHIE}

Confédération des syndicats nationaux (1996), Pour un développenent tourishique durable, p.64.

Le Groupe DBSF (1996), Diagnostic d'ensemble des ressources humaines en tourisme et plan d'action, p. 100 cité dans Confédération des syndicats nationaux, op.cit, p. 71.

Commission sur la fiscalité et le financement des services publics (1996), Ensemble pour un Québec responsable, Québec, p, 125.

Commission des droits de la personne du Qué. bec (1993) (1994), Les Enquetes à la Commis. sion des droits de la personne, Processus et résultats. 